\title{
La planificación como estrategia en las bibliotecas de la UPC
}

\author{
Por Dídac Martínez, Marta López-Vivancos, Sílvia Sunyer-Lázaro y Josep Vives-Gràcia
}

\begin{abstract}
Resumen: Bibliotecas con nuevos espacios y equipamientos para que los estudiantes de grado puedan trabajar y aprender en grupo, más contenidos personalizados de información electrónica como soporte al aprendizaje en red, depósitos digitales al servicio de la nueva publicación científica de la universidad para potenciar la visibilidad de la producción de los profesores e investigadores en internet, bibliotecarios que sepan idiomas, que asuman nuevos roles profesionales, que formen a los usuarios en las nuevas competencias transversales válidas para su vida profesional como son la habilidades informacionales. Estos son algunos de los ejes de Aprèn 2010, el $4^{\circ}$ plan estratégico de las bibliotecas de la UPC para el período 2007-2010, como respuesta de las bibliotecas a los nuevos retos europeos que la educación superior tiene planteados en los próximos años. Su lema: "Las bibliotecas de la UPC dan soporte al nuevo Espacio Europeo del Conocimiento".
\end{abstract}

Palabras clave: Planificación estratégica, Bibliotecas universitarias, Universitat Politècnica de Catalunya.

\section{Title: Strategic planning and the Technical Univer- sity of Catalonia (UPC) libraries}

Abstract: "Learn 2010," the fourth strategic plan implemented by the Technical University of Catalonia (UPC) libraries, outlines the libraries' response to the new challenges facing European higher education in the coming years. Its motto for 2007-2010 is: "The UPC libraries give support to the new European Space of Knowledge". The main goals of "Learn 2010" include: new library facilities and spaces that allow students to work and learn in groups, with innovative and personalized services, more electronic resources that meet user information needs and expectations in an online learning environment, digital academic repositories that support scientific publication and increase the visibility of faculty and researchers' academic output, and librarians with language skills who take on new professional challenges such as developing information literacy programs.

Keywords: Strategic planning, Academic libraries, Technical University of Catalonia

Martínez, Dídac; López-Vivancos, Marta; Sunyer-Lázaro, Sílvia; Vives-Gràcian, Josep. "La planificación como estrategia en las bibliotecas de la UPC”. En: El profesional de la información, 2007, julio-agosto, v. 16, n. 4, pp. $344-353$.

DOI: 10.3145/epi.2007.jul.08

\section{La planificación estratégica en el servicio de bibliotecas y documentación de la UPC}

LOS RESPONSABLES DE TODA ORGANIZACIÓN deben enfrentarse a dos tipos de decisiones: las tácticas, que hacen referencia al día a día de la institución y que tienen como objetivo prioritario solucionar problemas a corto plazo; $y$ las estratégicas, que tienen relación con la continuidad de la organización a lo largo de un tiempo, principalmente a medio y largo plazo, en tanto que identifican su ventaja competitiva en el mercado.

La toma de decisiones a medio y largo plazo dentro de una organización requiere de la planificación. Según Veciana (1999), planificar "consiste en trazar de antemano el camino a seguir a fin de dar congruencias al conjunto de acciones previstas, optimizar dicho conjunto y anticiparse al futuro y no dejarse dominar por él". Mediante la planificación estratégica las organizaciones definen sus políticas, su razón de ser (misión), sus aspiraciones (visión), sus valores, sus principales objetivos de futuro y proponen las acciones más adecuadas para alcanzarlos. 
"La UPC es pionera en

el ámbito universitario español en la introducción de la de la planificación estratégica como herramienta de gobierno y gestión de la universidad"

\subsection{La planificación estraté- gica en la $U P C$}

La Universitat Politècnica de Catalunya es pionera en el ámbito universitario español en la introducción de la de la planificación estratégica como herramienta de gobierno y gestión de la universidad. En un breve repaso a su reciente historia podemos destacar los siguientes planes estratégicos de los equipos de gobierno:

- Plan de futuro de la UPC 1990 encargado a la Unesco.

- Plan estratégico UPC 19941998. Misión: "calidad al servicio de la sociedad".

- Plan estratégico UPC 19982002. Misión: "una universidad emprendedora".

- Plan estratégico UPC 2003 2006. Misión: "excelencia académica, compromiso social, buen gobierno y atención a les personas".

- Plan estratégico UPC10 2007-2010. Misión: "calidad e innovación para un desarrollo sostenible de la sociedad".

http://www.upc.es/catala/laupc/planificacio/planificacio.htm

El actual plan estratégico se complementa con tres tipos de actuaciones estratégicas:

1. Contrato Programa de la $U P C$ con la Generalitat de Catalunya.

2. Marco para el impulso de las líneas estratégicas de las unidades básicas.
3. Planes transversales, de los cuales debemos destacar las siguientes:

- Plan estratégico de las bibliotecas de la UPC. Aprèn 2010.

- Plan estratégico del Servicio de Lenguas y Terminología.

- Plan de actuación tutorial.

- Plan de inversiones plurianual TIC.

- Plan UPC sostenible 2015.

- Plan de promoción de los estudios $U P C$.

- Plan de relaciones Internacionales de la $U P C$.

\subsection{Programas estratégicos del $S B D$}

El Servicio de Bibliotecas y Documentación $(S B D)$ de la UPC tiene también una reconocida y consolidada tradición en la gestión y la organización de sus bibliotecas mediante la planificación estratégica. Prueba de ello es que ha elaborado e implementado 4 planes estratégicos:

- Programa Leibniz 19911993. Misión: "mejorar las bibliotecas de la universidad".

- Programa Escher 19951999. Misión: "convertir las bibliotecas en un lugar para aprender a aprender".

- Programa Paideia 20002005. Misión: "elevar la calidad de la docencia e investigación de la UPC".

- Programa Aprèn 2007-2010. Misión: "dar soporte a los cambios que la universidad debe hacer frente dentro del horizonte 2010".

Todos ellos se consideran una herramienta transversal para la planificación y gestión de las diferentes bibliotecas de la universidad. Este modelo ha sido y es promovido por la propia dirección de la $U P C$ a lo largo de los últimos años. A continuación se exponen de for- ma resumida los resultados más significativos conseguidos por cada uno de ellos.

\section{3}

a. Programa Leibniz 1991-

- Creación del SBD.

- Instauración de un nuevo modelo organizativo: vicerrector de bibliotecas, comisión de bibliotecas de junta de gobierno, dirección, servicios técnicos, bibliotecas de centros.

- Elaboración de reglamentos y normas únicas.

- Creación y gestión de un presupuesto finalista.

- Política de adquisiciones de las bibliografías docentes.

- Creación del catálogo automatizado y único de la $U P C$.

- Implantación de la red bases de datos en cd-rom.

- Normalización de servicios bibliotecarios básicos para toda la comunidad.

- Profesionalización de la organización y creación de la plantilla de personal: jefes de biblioteca, bibliotecarios y auxiliares de biblioteca.

- Rehabilitación y creación de nuevas bibliotecas.

b. Programa Escher 1995-1999

- Incremento de las colecciones de soporte a la docencia.

- Aumento y nueva política de revistas científicas.

- Más desarrollo de las TIC en las bibliotecas.

- Creación de áreas de autoaprendizaje de idiomas, programas informáticos.

- Oferta de formación de usuarios.

- Mejora de todos los servicios bibliotecarios.

- Automatización de todos los procesos internos. 
- Incremento de la profesionalización del personal.

- Modificación del mapa bibliotecario: bibliotecas de campus.

- Inicio cooperación: $C B U C$ (Consorcio de Bibliotecas Universitarias de Catalunya).

\section{5}

c. Programa Paideia 2000-

- Nueva organización del $S B D$ por áreas: aprendizaje, investigación, digital y organización.

- Creación de Las Factorías como soporte a la innovación docente.

- Incremento de las bibliografías básicas y especializadas.

- Incremento de la información científica y técnica en soporte papel y electrónico.

- Nueva política de las colecciones de revistas en soporte papel y electrónicas.

- Nuevos servicios para los investigadores: Fenix.Doc, Observatorio de la producción científica.

- Creación de Bibliotècnica: la biblioteca digital de la $U P C$.

- Digitalización de colecciones y servicios a medida y online.

- Mejora de los procesos y servicios.

- Incremento de la cooperación y proyectos con $C B U C$, Rebiun, Ia- tul (International Association of Technological University Libraries).

- Profesionalización de las personas: perfiles, formación y comunicación.

- Evaluación y acreditación del SBD de Aneca, Aqu y Mec.

- Construcción de nuevas bibliotecas de campus y rehabilitación de espacios e instalaciones.

\section{Los retos de las universidades en el 2010}

2.1. El nuevo EEES y los nuevos métodos educativos

En el documento de la Comisión Europea El papel de las universidades en la Europa de conocimiento (2003) se apuntan los retos estratégicos a los que éstas deben hacer frente en los próximos años:

- El incremento de la demanda de formación superior, que provoca una saturación de su capacidad.

- La internacionalización de la educación y la investigación, fruto del impacto de las TIC, que se traduce en un aumento de la competencia entre las diferentes universidades y países.

- La multiplicación de los lugares de producción de conocimiento.

- La reorganización de los conocimientos, que se concreta en su diversificación, interdisciplinarie- dad y la aparición de nuevos campos de especialización.

- La aparición de nuevas expectativas a las que la universidad debe hacer frente, como sería el caso de asegurar la formación integral de los miembros de su comunidad.

Estos cinco retos se plasman de una manera objetiva en la Declaración de Bolonia (1999) que realizaron los ministros de educación de la Unión Europea y que persigue la construcción de un nuevo Espacio Europeo de Educación Superior (EEES).

Para construir el EEES resulta fundamental disponer de un sistema de titulaciones comprensible y comparable que facilite la movilidad; que sitúe al estudiante en el centro del proceso de aprendizaje; que estimule el desarrollo de la capacidad de aprendizaje a lo largo de la vida y que a medio plazo mejore la competitividad del sistema europeo de educación superior. El nuevo espacio se iniciaría en el año 2010 y tiene como hoja de ruta las diferentes actuaciones que debe realizar cada sistema universitario de los diferentes países de la UE.

Las actuaciones más significativas que cada universidad europea deberá implementar son:

- Sistema Europeo de Transferencia de Créditos (ECTS-European Credit Transfer System). Se

\section{Próximos temas centrales}

Septiembre 2007

Noviembre 2007

Enero 2008

Marzo 2008

Mayo 2008
Documentación multimedia Ontologías

Software libre para bibliotecas

Innovación en bibliotecas públicas

Presente y futuro de la profesión

Los interesados pueden remitir notas, artículos, propuestas, publicidad, comentarios, etc., sobre estos temas a:

epi@elprofesionaldelainformacion.com 
busca mejorar la comparación y transferencia de los cursos impartidos en cualquier estado europeo. Para calcular la duración de un crédito se enfatiza más el tiempo de estudio que debe dedicar el alumno en lugar del número de horas lectivas que recibe.

- Suplemento Europeo al Título. Es un documento que se anexa al título y que describe los estudios cursados para hacer posible una homologación y comparación a nivel europeo.

- Sistema de titulaciones de 2 ciclos. Partiendo del modelo anglosajón, las titulaciones consistirán en un primer ciclo de carácter genérico de 3-4 años de grado y un segundo ciclo de 1-2 años.

Por último cabe remarcar que el llamado proceso de Bolonia tendrá realmente sentido si las universidades realizan una profunda renovación del modelo educativo y de los métodos docentes. De poco servirán los cambios de convergencia europea si no van acompañados de una transformación pedagógica profunda.

\section{"De poco servirán los cambios de convergencia europea si no van acompañados de una transformación pedagógica profunda"}

El estudiante es el eje central de la universidad. Los nuevos modelos y prácticas pedagógicas deben tener en cuenta al alumno que aprende. Ése es uno de los objetivos de la aplicación de los nuevos créditos ECTS. Organizar e impartir asignaturas mediante créditos basados en la valoración del aprendizaje del estudiante y no en las horas lectivas que el profesor imparte.
Esta pequeña revolución es, desde nuestro punto de vista, la gran oportunidad de las bibliotecas para integrarse aún más en la mejora de la docencia de la universidad. En este contexto, las bibliotecas universitarias deben ser palancas del cambio con todos sus recursos y servicios. En el nuevo modelo educativo, los laboratorios, las prácticas en las empresas, la conexión a la Red y fundamentalmente el uso de la biblioteca tendrán un papel más relevante.

2.2. Las estrategias de las bibliotecas universitarias: el $\boldsymbol{C R A I}$

En este horizonte, la respuesta de las bibliotecas universitarias es la configuración e implementación de un nuevo modelo: la biblioteca renovada como un centro de recursos destinados para promover y dar soporte al nuevo aprendizaje: el Centro de Recursos para el Aprendizaje e Investigación (CRAI) (Martínez, 2003).

El objetivo de este modelo no es otro que adaptarse a las necesidades actuales y futuras de los estudiantes que quieren aprender. Podemos resumirlo en tres características:

"La gran sala de lectura de antaño se convierte ahora en una gran oferta de zonas diferenciadas donde los usuarios pueden elegir los espacios y los recursos según sus necesidades"

\section{a. Nuevos y diferenciados es- pacios}

Adaptar las zonas a las nuevas formas de estudio en grupo y de forma individual: instalaciones $y$ mobiliario adaptado no sólo para estudiar con los recursos de información clásicos (libros y revistas) y en silencio, ofreciendo lugares de estudio y trabajo diferenciado (salas de trabajo que se pueden reservar para trabajar en grupo, espacios de silencio para estudiar de forma individualizada, etc.). La gran sala de lectura de antaño se convierte ahora en una gran oferta de zonas diferenciadas donde los usuarios pueden elegir los espacios y los recursos según sus necesidades.

\section{b. Nuevas tecnologías educa- tivas}

La segunda característica es la variada oferta de tecnología educativa que el CRAI debe ofrecer a los usuarios: "information commons", salas multimedia, áreas con ordenadores de sobremesa, préstamo de portátiles, acceso a internet mediante la red sin cables, cibercafé, etc. (Leighton, 2003).

\section{c. Nuevas ofertas de servicios}

Muchas bibliotecas universitarias no ofrecen únicamente los servicios tradicionales sino que empiezan a poner a disposición de sus usuarios otros, como por ejemplo formación continuada del profesorado, enseñanza de idiomas, aprendizaje de búsqueda activa de trabajo, aprendizaje sobre técnicas de elaboración de proyectos, etc. Todos ellos siempre vinculados a la promoción y mejora del aprendizaje. Algunas bibliotecas, si los espacios e instalaciones lo permiten, han optado por ofrecer desde el $C R A I$ algunos servicios que ya existían en la universidad, como algunos de los relacionados con los servicios informáticos directos a los estudiantes, servicios de información general y de formación al profesorado, etc.

\subsection{La biblioteca digital como soporte al e-learning}

El elemento más significativo a nivel de recursos, colecciones y contenidos científicos y técnicos es, sin ninguna duda, la nueva biblioteca digital que se ofrece a los usuarios y especialmente a los in- 
vestigadores. En los últimos años las bibliotecas han procurado incrementar sus colecciones electrónicas con frecuentes adquisiciones, han aparecido nuevas formas y posibilidades de contratación y de gestión conjunta de grandes paquetes de información y hoy en día las bibliotecas digitales de las universidades poseen en líneas generales unos contenidos de gran calidad.

En una segunda etapa, que ahora se inicia, se han empezado a construir nuevos depósitos en donde se alojan los materiales educativos y trabajos académicos, no sólo para dar soporte al aprendizaje distribuido (e-learning) sino para aumentar la visibilidad de la producción científica de los investigadores en internet. La personalización de los servicios en línea es otra de las características de la nueva biblioteca digital juntamente con la socialización de algunos de sus apartados: la biblioteca digital 2.0.

\section{Aprèn 2010}

3.1. Metodología del plan estratégico

Aprèn 2010 no es un plan estratégico elaborado en un laboratorio de ideas, sino que es el resultado de la reflexión de las personas que integran las bibliotecas de la UPC. Desde el primer momento se consideró que era necesario contar con la participación de todos los bibliotecarios y técnicos de nuestro servicio. Es cierto que esto ha conllevado más trabajo de coordinación

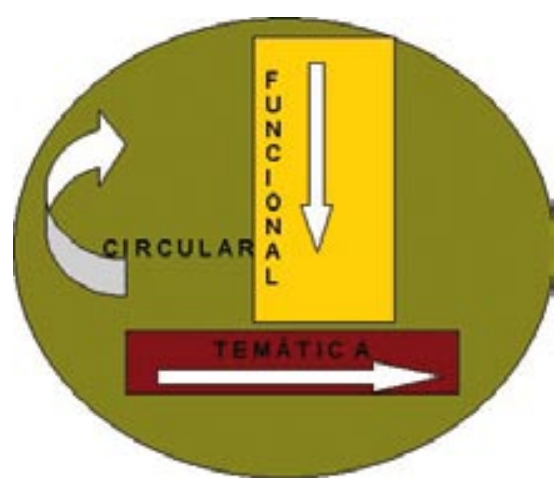

Figura 1 y planificación (reuniones, elaboración de documentos, puesta en común, etc.) pero se consideró que este sobreesfuerzo de redacción estaría compensado con el resultado de un plan estratégico que reflejara las aspiraciones del mayor número posible de personas. A estos efectos, su elaboración se organizó desde una perspectiva tridimensional: funcional, temática y circular.

Con el fin de poder disponer de diferentes puntos de vistas, se constituyeron tres grupos de trabajo:

- Dirección: formado por la dirección del $S B D$, los jefes de unidad, jefes de bibliotecas y responsables de los servicios para el aprendizaje, servicios digitales y servicios para la investigación.

- Análisis y estudio de temas clave: se crearon diecisiete grupos de análisis temáticos a partir de una serie de temas que se consideraron claves para el futuro de las bibliotecas.

- Redacción: formado por la dirección del servicio de biblioteca, una jefa de biblioteca y dos bibliotecarios. Su misión sería la de elaborar el borrador del plan estratégico que tendría que contener los principales puntos incluidos en los análisis de los dos anteriores grupos.

El grupo de dirección (visión vertical o funcional) era el responsable de la elaboración de los diferentes estudios $D A F O$ a partir de la reunión del personal de las bibliotecas y de los servicios generales. Así pues, cada biblioteca y servicio participó en la elaboración del plan estratégico redactando las conclusiones de su propio análisis. En total se presentaron 16 análisis $D A F O, 12$ correspondientes a cada una de las bibliotecas de la UPC y 3 provenientes de los servicios centrales existentes (servicios para el aprendizaje, para la investigación y digitales). El personal de las bibliotecas se reunió para realizar su propio análisis. Posteriormente, se presentaron las conclusiones del análisis estratégico agrupando los diferentes ítems en:

- Puntos débiles/fuertes.

- Amenazas/oportunidades.

- Propuesta de líneas estratégicas para la biblioteca.

- Propuesta de líneas estratégicas para el servicio en general.

Estos 16 análisis daban una buena visión de la situación actual y las aspiraciones futuras desde la perspectiva de servicio. Un ejemplo de una parte del análisis efectuado por cada biblioteca y servicio general se muestra en la figura 2.

Para complementar esta visión funcional, se crearon también 17 ámbitos temáticos cuya misión era la de elaborar un estado de la cuestión en algunos de las temas clave que influyen actualmente las bibliotecas. El personal bibliotecario podía participar en un máximo de dos grupos de trabajo temáticos y cada grupo elaboró un documento en el que se analizaba el tema asignado y cómo debía ser abordado por la biblioteca.

Es necesario decir que tanto los grupos temáticos como los funcionales (por bibliotecas o áreas) disponían de total libertad para decidir qué cuestiones eran las que cabía señalar. Dicho de otra forma, lo que se quería conseguir era que se dejara constancia de los aspectos favorables pero muy especialmente que también aparecieran los aspectos negativos de la organización. De poco sirve un análisis $D A F O$ que se limite a alabar lo propio.

Aunque pueda resultar prolijo, se considera de alto interés citar los ámbitos temáticos que se tuvieron en cuenta y que dieron la visión transversal al proyecto:

- La biblioteca como CRAI: servicios integrados y nuevos equipamientos. 


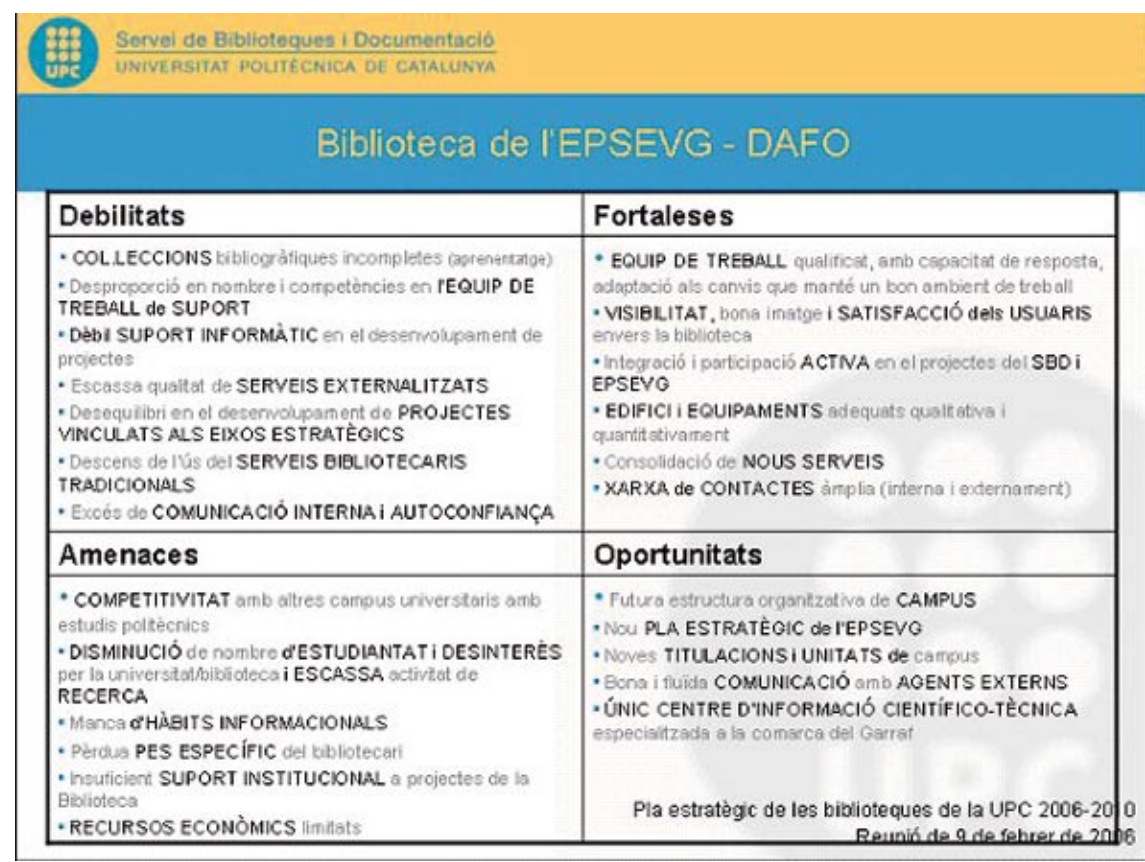

Figura 2

- La formación de usuarios y las habilidades informacionales.

- Las nuevas políticas de gestión de las colecciones.

- Los derechos de autor en la biblioteca digital.

- Las relaciones externas y la cooperación en red de las bibliotecas universitarias.

- La gestión y administración de los recursos: eficiencia y rendición de cuentas.

- Las nuevas competencias de los bibliotecarios y los técnicos de las bibliotecas.

- El apoyo de las bibliotecas a los nuevos retos del EEES.

- La investigación en la UPC y los nuevos servicios bibliotecarios.

- La calidad y la evaluación continuada de los servicios.

- La Factoría de Recursos Docentes y otros servicios de apoyo a la innovación docente y el e-learning.

- Análisis y estudio de otros planes estratégicos de bibliotecas universitarias.

Finalmente, el grupo de redacción tenía como misión dar forma ma europeo de educación superior en los próximos años y la necesidad de la implicación de la misma Comisión Europea en la mejora de nuestra universidad. La homologación de las titulaciones, la mejora de la calidad docente, el impulso de la investigación o la imbricación universidad-sociedad son sus puntos más destacados. En esta misma idea de desarrollar la idea de "espacio de conocimiento", el gobierno catalán igualmente aprobó en 2004 la creación de la Oficina de l'Espai Europeu del Coneixement. La idea subyacente es la necesidad de desarrollar tanto una transformación de la educación superior (Espacio Europeo de Educación Superior) como, y al mismo tiempo, dar impulso a la investigación que se lleva a cabo en las universidades (Espacio Europeo de Investigación). Es necesario insistir en la bicefalia de los objetivos (docencia+investigación) pues frecuentemente las referencias al impulso de la investigación en la universidad han quedado difuminadas por el debate sobre la mejora y adaptación de los estudios universitarios. La idea de espacio europeo del conocimiento recoge a nuestro entender los dos procesos necesarios.

http://www10.gencat.net/dursi/ ca/de/eec_oeec.htm

\section{a. Misión de las bibliotecas de la UPC en Aprèn 2010}

Las bibliotecas, en tanto que son entornos ricos en información, tecnología y personas, deben integrarse en las actividades de docencia, aprendizaje e investigación de la UPC para contribuir al logro de los nuevos retos del espacio europeo del conocimiento.

La formulación de la misión en estos términos huye de conceptos clásicos como "gestionar colecciones", "ser depositarios" o "facilitar servicios a nuestra comunidad". Esta huida es consciente y voluntaria ya que lo se busca es la implicación de las bibliotecas en los 
objetivos generales de la universidad dentro de sus funciones. Lo que habitualmente se conoce como el proceso de Bolonia debe ser un reto no solamente para la universidad (órganos de gobierno y docentes) sin también para todas las personas de nuestro servicio. La voluntad de la misión es implicar todavía más a las bibliotecas de la UPC en el desarrollo normal de las funciones de la universidad.

\section{"La voluntad es implicar todavía más a las bibliotecas de la UPC en el desarrollo normal de las funciones de la universidad"}

Se entiende que la misión del $S B D$ es colaborar con la institución a la que se presta servicio en la transmisión y generación de conocimiento útil para la sociedad. Como se afirma en The academic library in 2010: a vision (AUL, 2005): "these are the best of times for libraries. People are hungry for information at the same time they are inundated with it. In the midst of information overload, students are looking for meaning and knowledge".

Paralelamente a la formulación de la misión, se desarrolló como procede, una visión que marcase cuál debería ser el nivel de desarrollo de la misión. La visión escogida desarrolla y concreta alguno de los aspectos de la misión anteriormente enunciada.

\section{b. Visión para las bibliotecas de la UPC en Aprèn 2010}

Devenir centros activos de recursos y servicios de calidad para ofrecer respuesta a medida de las necesidades de aprendizaje, investigación y formación continua de la comunidad universitaria. Esto se pretende conseguir:
- Ofreciendo espacios versátiles, con un alto componente tecnológico, que favorezcan el logro de los retos que la educación superior debe asumir en 2010.

- Colaborando estrechamente con las diferentes unidades de la $U P C$ y otras organizaciones externas.

- Implicando a las bibliotecas de manera activa en el proceso de aprendizaje, investigación y formación continuada que la comunidad universitaria desarrolla en la $U P C$.

- Convirtiendo Bibliotècnica, la biblioteca digital de la $U P C$, en un portal de recursos y servicios personalizados.

- Dando a conocer de forma eficaz los recursos y servicios de las bibliotecas.

- Consolidando una estructura organizativa orientada al usuario y basada en criterios de calidad.

La visión en los términos propuestos parte de una actitud proactiva de las bibliotecas de la UPC y se formula marcando una primera serie de objetivos a cumplir. Si se quisiera señalar en forma de palabras clave la voluntad que se expresa en el desarrollo de nuestro punto de vista se hablaría de: espacios, colaboración, biblioteca digital o difusión.

Naturalmente, no se podía obviar la necesaria transición de la biblioteca de papel a la electrónica. Exactamente no se conoce todavía como será pero podemos estar seguros que existirá. Este cambio afectará sin lugar a dudas a los servicios (qué ofreceremos y cómo) y por supuesto al perfil de los profe- sionales que actualmente dan servicios en las bibliotecas (Lewis, 2007).

Finalmente, el plan estratégico incorpora una nueva forma de presentación de los valores corporativos ante la comunidad de usuarios. La tendencia en el desarrollo de valores corporativos es un hecho cada vez más frecuente en la planificación estratégica de las organizaciones, especialmente si en éstas desarrollan su actividad profesional un considerable número de personas. Algunos autores señalan que cerca del $50 \%$ de las organizaciones disponen de algún tipo de reflexión al respecto (Jaakson, 2004). Dichos valores deben ser comunicados e incorporados en las actividades de la institución de forma que las personas puedan identificarse y actuar de acuerdo a ellos.

http://www.teal.org.uk/mat/ page6.htm

Los valores corporativos representan aquellos aspectos con los que las organizaciones desean ser identificados; así, algunas bibliotecas como la British Library hablan de que éstos señalan la "manera de hacer" con la que la organización quiere ser vista.

http://www.bl.uk/about/strategic/pdf/blstrategy20052008.pdf

La defensa de valores corporativos como bien común, democracia, igualdad, ética, derechos humanos, etc. se puede encontrar en las definiciones de, por ejemplo, muchas bibliotecas públicas danesas (Johannsen, 2004). Dichos valores pueden hacer referencia a principios generales que definen en muchos casos el marco de trabajo institucional

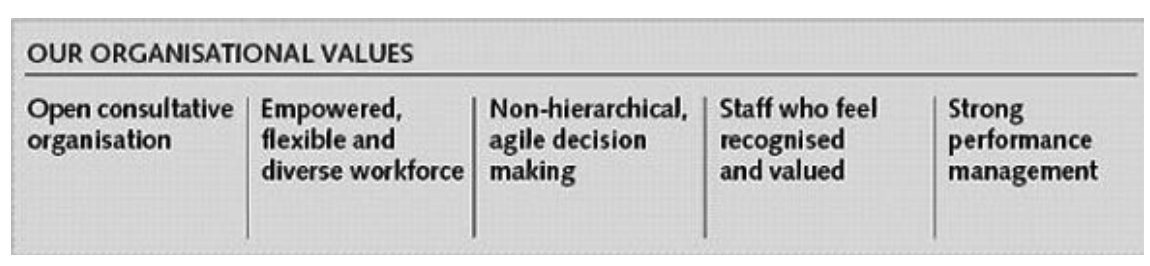

Figura 3 
de una biblioteca pero igualmente pueden señalar actitudes determinadas con las que las personas de la organización se identifican en su quehacer tales como eficiencia, entusiasmo, flexibilidad, profesionales, etc.

La mención de valores corporativos se suele hacer explícitamente en los planes estratégicos de la organización de forma paralela a la definición de la misión y visión. Así por ejemplo, la biblioteca de la Universidad de Sidney, los coloca en el primer lugar en la redacción de su plan estratégico, una forma sencilla de transmitir, de humanizar (si se quiere) a la institución.

http://www.library.usyd.edu. au/about/strategicplan/LibrarysStrategicPlan.pdf

En el caso de Aprèn2010 se consideró muy oportuno definir una serie de valores que reflejaran la voluntad colectiva del Servei de Biblioteques $i$ Documentació de la $U P C$. Fueron redactados por el equipo de redacción y presentados a consulta junto con la resta del borrador de plan estratégico:

- Calidad.

- Accesibilidad.

- Sostenibilidad.

- Cooperación.

- Lealtad.

- Tecnología.

- Visibilidad.

- Multilingüismo.

- Solidaridad.

- Comunicación.

- Acceso abierto.

- Multiculturalidad.

- Profesionalidad.

Cada uno de ellos se comunica a través de un breve lema de forma que se clarifica su contexto: "Acceso abierto: daremos apoyo a las iniciativas para compartir el conoci- miento científico y técnico mediante el establecimiento de políticas de acceso abierto a la información".

La selección respondió a diferentes criterios. Unas veces se recogían los de la propia universidad (sostenibilidad, cooperación, tecnología, etc.), valores sociales (multiculturalidad, solidaridad, multilingüismo) y también actitudes que las bibliotecas de la UPC han ido haciendo suyas en el transcurso de los años (profesionalidad, visibilidad, acceso abierto, etc.).

\subsection{Ejes, áreas de actuación e indicadores}

Se estructura en 10 ejes que a su vez se subdividen en 5 áreas de actuación, con un total de 50 áreas a desarrollar en los próximos años. Los 10 ejes de actuación aglutinan aquellos conceptos que se consideran claves para el desarrollo de las bibliotecas durante el período 2007-2010. Para cada uno de ellos se ha establecido una batería de indicadores, cuantitativos y cualitativos, que tienen que permitir medir el nivel de logro de las actuaciones propuestas.

\section{a. Ejes de actuación}

- Instalaciones.

- Tecnología.

- Colecciones.

- Bibliotècnica: la biblioteca digital de la $U P C$.

- Servicios bibliotecarios.

- Habilidades informacionales.

- Conocimiento UPC.

- Acceso a la cultura.

- Profesionales.

- Organización y gestión.

Naturalmente, se considera que todos son importantes, empezando por el primero que incide en la mejora de las instalaciones, espacios y equipamientos de las bibliotecas con el fin de dar soporte a las nuevas formas de estudio, aprendizaje e investigación, o el que hace referencia a la biblioteca digital de la $U P C$ que quiere consolidarse como un espacio de acceso a contenidos y servicios personalizados de calidad y de soporte a los campus virtuales.

Pero para las bibliotecas de la $U P C$ hay dos especialmente estratégicos y en los cuales se centrarán los esfuerzos de los profesionales: se trata del desarrollo del programa de formación en habilidades informacionales y el incremento y consolidación del portal UPCommons, que aglutina todos los depósitos institucionales abiertos de la $U P C$.

\section{https://upcommons.upc.edu/}

De todos es conocido que la adquisición de competencias informacionales es una tarea a desarrollar en los nuevos planes de estudios adaptados al EEES en los cuales los profesionales de las bibliotecas pueden (y deben) colaborar de forma activa. El servicio de bibliotecas de la $U P C$, con una dilatada experiencia en la impartición de formación vinculada al conocimiento $\mathrm{y}$ la mejora del uso de los servicios y recursos de información (García, 2006), ha elaborado una propuesta de implementación de una asignatura de habilitades informacionales en los estudios de grado y postgrado de la UPC. Durante los próximos cursos las bibliotecas continuarán desarrollando las estrategias para conseguir incluir en los planes de estudio politécnicos adaptados al EEES las actividades formativas que han de permitir desarrollar las competencias transversales relacionadas con la búsqueda efectiva y el acceso a la información.

Otro de los ejes de actuación más destacados es el que hace referencia al denominado conocimiento UPC. El Servicio de Bibliotecas y Documentación lidera iniciativas para dar a conocer el movimiento de libre acceso a las publicaciones 
científicas y proporcionar a los autores de la universidad herramientas que faciliten la publicación de sus resultados académicos, potenciando así el acceso abierto y también su preservación. Las diferentes iniciativas de las bibliotecas se sintetizan en UPCommons que facilita el acceso unificado a los distintos depósitos institucionales estructurados según la tipología de documentos: e-prints, revistas, tesis, proyectos y trabajos de final de carrera, materiales docentes, etc. Durante los próximos años se trabajará para aumentar el número de recursos disponibles con el fin de incrementar la visibilidad, el impacto y el prestigio de los autores, así como de la propia universidad.

\section{Modelo de gestión del plan estratégico}

\subsection{Definición y gestión de los objetivos específicos}

Tomando como marco de referencia las directrices generales establecidas en los distintos planes estratégicos, la dirección del $S B D$ y las 12 bibliotecas que integran el servicio establecen programas de acciones anuales. Es un proceso sistematizado que fija objetivos con plazos y fechas, que designa recursos y que asigna al personal su cometido.

Se trata de movilizar los esfuerzos concretos de las bibliotecas de la $U P C$ al logro de resultados de valor, siempre desde la perspectiva de la orientación al usuario.

El establecimiento de los objetivos se basa en el trabajo en grupo, en el concepto de servicio único y en la participación de todos los profesionales de las bibliotecas y también de los agentes de la universidad. La clara orientación al cambio e innovación de las bibliotecas de la UPC sirve de impulso para aprovechar o crear oportunidades, así como asumir responsabilidades en la toma de decisiones.

\subsection{Seguimiento y evaluación}

Con todo, la planificación, la fijación de unas metas y objetivos, así como el desarrollo de los planes necesarios para alcanzarlos, puede no llevar a resultados óptimos si su ejecución no se basa en criterios de calidad y no es objeto de un control para aplicar pautas de mejora. Por este motivo, y así lo recoge también Aprèn 2010, las bibliotecas han desarrollado estrategias y herramientas de gestión concretas, como una intranet para la gestión de documentos, una base de datos de objetivos anuales, wikis (sitios web colaborativos que pueden ser editados por varios usuarios) o un exhaustivo almacén de datos estadísticos de las bibliotecas ( $D a$ tawarehouse) que da soporte en la toma de decisiones. Próximamente se quiere iniciar la revisión y mejora de los indicadores de calidad, especialmente analizar la posibilidad de establecer algunos no estrictamente cuantitativos.

\section{Conclusiones}

Haciendo un paralelismo con la educación secundaria, podríamos afirmar que las universidades europeas se enfrentan ahora a su propia Logse. Los principios que propugnaban la reforma pedagógica en la enseñanza secundaria parece que son los mismos que impregnan los nuevos aires de cambio en la educación superior. En su momento, sin embargo, la Logse fracasó en ciertos aspectos debido a una falta de planificación, recursos, y, seguramente falta de voluntad de los

\section{"Haciendo un paralelismo con la educación secundaria, podríamos afirmar que las universidades europeas se enfrentan ahora a su propia Logse"}

actores implicados. Algo parecido podría acontecer con la reforma universitaria que impulsa la Unión Europea si todos los agentes involucrados en la educación superior no trabajan aunando esfuerzos, recursos y conocimientos.

\section{El Servicio de Bibliotecas y} Documentación de la UPC es consciente de la importancia que para el futuro de su universidad y de Europa tiene alcanzar tanto el Espacio Europeo de Educación Superior como el Espacio Europeo de Investigación.

A partir de esta premisa, el $S B D$ ha elaborado su plan estratégico que plasma su compromiso con la consecución de unos objetivos determinados. Aprèn 2010 es el marco bajo el cual el $S B D$ se compromete a poner todo su empeño en colaborar en los retos que tiene planteada la universidad. La realización de Aprèn 2010 ha sido el fruto del trabajo colectivo de las personas que forman parte de las bibliotecas de la $U P C$, y a partir de una triple visión (funcional, temática y circular), se han ido definiendo los aspectos estratégicos como la misión, la visión, los valores y finalmente los objetivos para el período 2007-2010.

\section{Bibliografía}

American University Library. "The academic library in 2010: a vision”. En: Report of symposium 2010. Washington, DC March 14-15, 2005. Consultado en: 16-03-07.

http://www.library.american.edu/Symposium 2010.pdf

Comisión de las Comunidades Europeas. El papel de las universidades en la Europa del conocimiento: comunicación de la Comisión. 5/02/2003. COM(2003). Consultado en: 16-03-07.

http://eees.universia.es/historia/papel-universidades/com2003-0058es01El-papel-de-las-univen-la-Europa-del-conoc.pdf

Comisión de Cultura, Juventud, Educación, Medios de Comunicación y Deporte del Parlamento Europeo. Informe sobre las universidades y la enseñanza superior en el espacio europeo del conocimiento. 24 mayo 2002. Consultado en: 16-03-07.

http://www.uam.es/europea/antiguaEEES/parlamentoeuropeo.pdf

El Espacio Europeo de la Enseñanza Superior: declaración conjunta de los ministros europeos 
